\title{
Direct Astrocytic Contacts Regulate Local Maturation of Dendritic Spines
}

\author{
Hideko Nishida ${ }^{1,2}$ and Shigeo Okabe ${ }^{1,2,3,4}$ \\ ${ }^{1}$ Department of Cell Biology, School of Medicine, and ${ }^{2} \mathrm{COE}$ Program for Brain Integration and its Disorders, Tokyo Medical and Dental University, Tokyo \\ 113-8519, Japan, ${ }^{3}$ Solution Oriented Research for Science and Technology, Japan Science and Technology Agency, Kawaguchi 332-0012, Japan, and \\ ${ }^{4}$ Molecular Neurophysiology Group, Neuroscience Research Institute, National Institute of Advanced Industrial Science and Technology, Ibaraki 305-8566, \\ Japan
}

Astrocytes contribute on both development and function of synapses, but it remains unclear whether direct astrocytic contacts regulate development of individual synapses. Two-photon time-lapse imaging of astrocytic and dendritic protrusive activity revealed the correlation of astrocytic contacts with both lifetime and morphological maturation of dendritic protrusions. Astrocytic motility was essential in maturation of spines, because its suppression by manipulating Rac1-dependent signaling in astrocytes resulted in induction of longer, filopodia-like dendritic protrusions. Manipulation of ephrin/Eph-dependent neuron-astrocyte signaling suggested involvement of this signaling pathway in astrocyte-dependent stabilization of newly generated dendritic protrusions. Our data support a model in which astrocytic protrusive activity in development acts as a key local regulator for stabilization of individual dendritic protrusions and subsequent maturation into spines.

Key words: dendritic spine; astrocyte; organotypic slice; two-photon microscopy; motility; synapse

\section{Introduction}

Increasing evidence indicates the importance of neuron-astrocyte interaction in synaptic development and function (Haydon, 2001; Ullian et al., 2001; Hansson and Rönnbäck, 2003; Slezak and Pfrieger, 2003; Schipke and Kettenmann, 2004; Wang et al., 2006). Astrocytes secrete diffusible factors, such as cholesterol (Mauch et al., 2001), tumor necrosis factor- $\alpha$ (Beattie et al., 2002), activity-dependent neurotrophic factor (Blondel et al., 2000), and thrombospondins (Christopherson et al., 2005), which can affect both nearby and remote neurons to promote their synapse formation. Direct astrocytic contacts can also induce global maturation of neurons and subsequently upregulate synapse formation in a protein kinase C-dependent manner (Hama et al., 2004). These studies indicate the importance of astrocytes in promoting global maturation of neurons and their network.

Astrocytic contacts may induce local structural and functional modifications of dendritic segments or individual synapses. In the mature nervous tissue, this contention has been supported by several evidences. An electron microscopic reconstruction study

Received Oct. 13, 2006; revised Dec. 2, 2006; accepted Dec. 4, 2006.

This work was supported by grants from the Ministry of Education, Culture, Sports, Science, and Technology of Japan, the Japan Society for the Promotion of Science, and the Japan Science and Technology Corporation. We thank H. Okado for recombinant adenovirus vectors (Ad-Cre and Ad-loxP-GFP), M. Enomoto and T. Urushido for a bicistronic recombinant adenovirus vector [Ad-Rac1(N17)-IRES-GFP], R. Shigemoto and A. Lörincz for a technical lesson of three-dimensional reconstruction from ultrastructural images, and Y. Kubo for valuable advice.

Correspondence should be addressed to Dr. Shigeo Okabe, Department of Cell Biology, School of Medicine, Tokyo Medical and Dental University, 1-5-45, Yushima, Bunkyo-ku, Tokyo 113-8519, Japan. E-mail: okabe.cbio@tmd.ac.jp.

DOI:10.1523/JNEUROSCI.4466-06.2007

Copyright $\odot 2007$ Society for Neuroscience $\quad$ 0270-6474/07/270331-10\$15.00/0 revealed that $57 \%$ of synapses in the mature hippocampus are in direct contact with astrocytes (Ventura and Harris, 1999). Membrane-bound ligands on the astrocytes, such as ephrin-A3, have been shown to regulate spine morphology in the hippocampus (Murai et al., 2003), suggesting local activation of EphA receptors on spines by astrocytic ephrin-A3. Furthermore, a recent time-lapse imaging showed cooperative astrocyte-spine motility over the course of minutes in mature hippocampal slices (Haber et al., 2006).

Astrocytic contacts may have distinct roles in initial synapse formation. Dendritic protrusive activity is prominent in developing neurons (Dailey and Smith, 1996; Ziv and Smith, 1996; Portera-Cailliau et al., 2003). Based on the imaging studies of cultured neurons, it has been postulated that a subset of motile protrusions are selectively stabilized and transformed into stable spines with synaptic contacts (Yuste and Bonhoeffer, 2004). Electron microscopic studies provided evidences supporting another model, in which initially developed shaft synapses are later transformed into spine synapses without losing synaptic contacts (Fiala et al., 1998; Yuste and Bonhoeffer, 2004). A recent in vivo imaging study with electron microscopic reconstruction of imaged spines revealed spontaneous appearance of spines without synaptic contacts with axon terminals (Knott et al., 2006). This observation is consistent with the former hypothesis, namely "filopodia model" of synaptogenesis. These arguments illustrate the importance of selective stabilization of a subset of dendritic protrusions in the early phase of synapse development. Local astrocytic contacts may regulate stabilization and maturation of dendritic protrusions even before their formation of synaptic contacts. There have been very few reports, however, of studies 
designed to explore the contact-mediated regulation of synapse/ spine development by astrocytes.

Here we performed two-photon imaging of astrocytic processes and dendritic protrusions in developing hippocampal tissue slices. Astrocytic contacts enhanced both lifetime and morphological maturation of dendritic protrusions. Suppression of astrocytic motility by manipulating Racl-dependent signaling resulted in induction of filopodia-like immature dendritic protrusions. Acute blockade of ephrin/Eph-dependent neuron-astrocyte signaling destabilized newly generated dendritic protrusions. Our data collectively support a model in which protrusive activity of astrocytes critically regulates both stabilization of individual dendritic protrusions and their subsequent maturation into spines.

\section{Materials and Methods Preparation of hippocampal slice cultures}

Hippocampal slice cultures from postnatal day 4-5 mice were prepared as described previously (Stoppini et al., 1991; Umeda et al., 2005). Briefly, the dissected hippocampus and cortex were cut into $375 \mu \mathrm{m}$ slices with a McIlwain-type tissue chopper. Slices were placed on transparent porous filters (Millicell CM; Millipore, Bedford, MA) in a $34^{\circ} \mathrm{C}, 5 \% \mathrm{CO}_{2}$ humid incubator and fed every $3 \mathrm{~d}$ with culture medium containing $48 \%$ Eagle's basal medium, 24\% Earle's balanced salt solution, and 24\% horse serum supplemented with $5 \mathrm{mg} / \mathrm{ml}$ glucose, $10 \mathrm{~mm}$ HEPES, $1 \mathrm{~mm}$ L-glutamine, $50 \mathrm{U} / \mathrm{ml}$ penicillin, and $50 \mathrm{U} / \mathrm{ml}$ streptomycin.

\section{Visualization of astrocytes and pyramidal neurons in hippocampal slice cultures}

Two types of recombinant adenoviruses were used. One was for the expression of Cre recombinase (Ad-Cre), and the second was for the expression of green fluorescent protein (GFP) under the CAG promoter with intervening loxP sites and stuffer sequences (Ad-loxP-GFP). After coinfection of the two adenoviruses, the stuffer sequences were excised, and GFP expression was initiated. The number of plaque-forming units (PFUs) of Ad-Cre was adjusted to 1:10,000 of Ad-loxP-GFP to restrict the expression of GFP in a small subset of cells. These two adenoviruses were injected together to slices $2 \mathrm{~d}$ before imaging sessions of astrocytes. To characterize the GFP-positive cells and to confirm the distribution of GFAP-positive cells in slice cultures, immunohistochemistry using antiGFAP antibody [rabbit polyclonal (1:200; Zymed, Carton Court, CA) and mouse monoclonal (1:500; Sigma, St. Louis, MO)] was performed.

Rhodamine-dextran (TMRD; Invitrogen, Eugene, OR) was electroporated into a single pyramidal cell as described previously (Haas et al., 2001; Umeda et al., 2005). In brief, slice cultures were placed in a $10 \mathrm{~cm}$ dish containing low-serum medium (48\% Eagle's basal medium, $43 \%$ Earle's balanced salt solution, $5 \%$ horse serum, $5 \mathrm{mg} / \mathrm{ml}$ glucose, $10 \mathrm{~mm}$ HEPES, $1 \mathrm{~mm} \mathrm{~L}$-glutamine, $50 \mathrm{U} / \mathrm{ml}$ penicillin, and $50 \mathrm{U} / \mathrm{ml}$ streptomycin) on the microscope stage. A micropipette containing $8 \mathrm{mg} / \mathrm{ml}$ rhodamine-dextran was placed just above the cell body of a pyramidal neuron using a $60 \times$ water-immersion lens, and 200 rectangular pulses $(30 \mathrm{~V}$, 1 ms duration) were applied at $200 \mathrm{~Hz}$.

\section{Mutant Racl and recombinant adenovirus}

Recombinant adenoviruses expressing a dominant-negative form of Racl (N17), together with a marker gene GFP under the control of CAG promoter, were constructed as described previously using the Adenovirus Expression Vector kit (TaKaRa, New York, NY) (Okabe et al., 1999). By using the internal ribosomal entry site-based bicistronic vector system, both GFP and a mutant form of Rac1 were expressed by infection of a single construct of adenovirus. Recombinant adenoviruses expressing mutant Rac1 (N17) were applied to the slice $2 \mathrm{~d}$ before observation.

\section{EphA4/Fc and ephrin-A3/Fc reagents}

EphA4/Fc and ephrin-A3/Fc, fusions of the Fc domain of the human IgG1 with EphA4 and ephin-A3, were from R \& D Systems (Minneapolis,
$\mathrm{MN}$ ), applied at the concentration of $6.7 \mu \mathrm{g} / \mathrm{ml}$ and incubated for $30 \mathrm{~min}$ before imaging.

\section{Imaging}

Live hippocampal slice cultures (3-7 d in culture) were mounted in a chamber containing low-serum medium. The temperature of medium was kept at $30-31^{\circ} \mathrm{C}$. Paraffin oil was overlaid on the surface of medium to prevent evaporation.

Simultaneous imaging of GFP and rhodamine-dextran was performed with two-photon excitation at $860 \mathrm{~nm}$ (R2000MP; Bio-Rad, Hercules, CA) with a $60 \times$ water-immersion lens. Images were collected at additional digital zooms resulting in a nominal spatial resolution of 62 $\mathrm{nm}$ per pixel. Multiple optical sections $(7.4-12.4 \times 16 \mu \mathrm{m}$ cross sections scanned 8-14 times with z-spacing of $0.75 \mu \mathrm{m})$ were collected in both areas CA1 and CA 3 every $30 \mathrm{~min}$ for $>5.5 \mathrm{~h}$.

\section{Data analysis}

Fluorescent objects extending from dendritic shafts with their length in the range of $0.3-5 \mu \mathrm{m}$ were judged as dendritic protrusions. Their morphology was variable; spines were defined as protrusions with bulbous heads and neck constrictions. Protrusions not classified as spines, namely protrusions without bulbous heads, were heterogeneous, including those referred to as filopodia-like or stubby protrusions. Dendritic protrusions were considered to be the same protrusion when they were present at the same spatial point on the dendritic shafts between frames. The length and area of each dendritic protrusion (see Figs. $3 j, k, 5 e, f$ ) and the length of astrocytic processes (branches with their length in the range of $0.3-5 \mu \mathrm{m}$ ) (see Fig. $5 e$ ) were measured manually by using the MetaMorph software (Universal Imaging, West Chester, PA).

The lifetime (see Figs. $3 f, 4 d, 6 c, d)(<0.5-5.5 \mathrm{~h})$ was determined for all dendritic protrusions by calculating $(n-1) \times 0.5 \mathrm{~h}$, where $n$ is the number of frames showing continuous presence of protrusions (if $n=1$, lifetime was estimated to be $0.25 \mathrm{~h}$ ). Dendritic protrusions were considered to have disappeared when they retracted to the length of $<0.3 \mu \mathrm{m}$.

From merged images of dendritic protrusions (rhodamine-dextran) and astrocytic processes (GFP), their contact events were investigated. Contact events were defined as an overlap of pixels derived from two structural components within a single optical section. Contacts consecutive in multiple time frames were considered to be a single contact event. Dendritic protrusions without any astrocytic contact episodes throughout observation were analyzed when the distance to the nearest astrocytic process was $<3 \mu \mathrm{m}$. The actual mean distance was $1.42 \pm 0.24$ $\mu \mathrm{m}$ (mean $\pm \mathrm{SEM} ; n=12$ dendritic protrusions).

All imaged protrusions were classified into four groups: spines with astrocytic contacts $(\mathrm{S}+\mathrm{A})$, spines without astrocytic contacts $(\mathrm{S})$, dendritic protrusions without bulbous heads but with astrocytic contacts (NS + A), and dendritic protrusions having neither bulbous heads nor astrocytic contacts (NS). We created a table of the temporal transition of individual protrusions by classifying them into corresponding four groups and calculated the probability of disappearance and spine formation.

Contact index (see Fig. $3 g$ ) was a proportion of the time frames showing contacts between dendritic protrusions and astrocytes to the time frames within a period of $1 \mathrm{~h}$ before disappearance of the dendritic protrusion. Randomly sampled consecutive time frames of $1 \mathrm{~h}$ duration were used as control.

To calculate the probability of protrusion disappearance (see Fig. $3 h$ ), all two consecutive time frames showing all types of structural transition (any transitions among four groups and protrusion disappearances) were extracted. From the number of all types of structural transitions $(j)$ and that of protrusion disappearance $(k)$, the probability of protrusion disappearance was calculated as $k / j$.

To calculate the probability to form spines from NS or NS+A (see Figs. $3 i, 6 f$ ), all two consecutive time frames showing all types of structural transition from NS or NS+A (any transitions from NS or NS+A into other groups and protrusion disappearance) were extracted and counted $(l)$. From this number and the number of transitions from NS or NS + A into $\mathrm{S}+\mathrm{A}$ or $\mathrm{S}(\mathrm{m})$, the probability to form spines from NS and NS+A was calculated as $m / l$. 

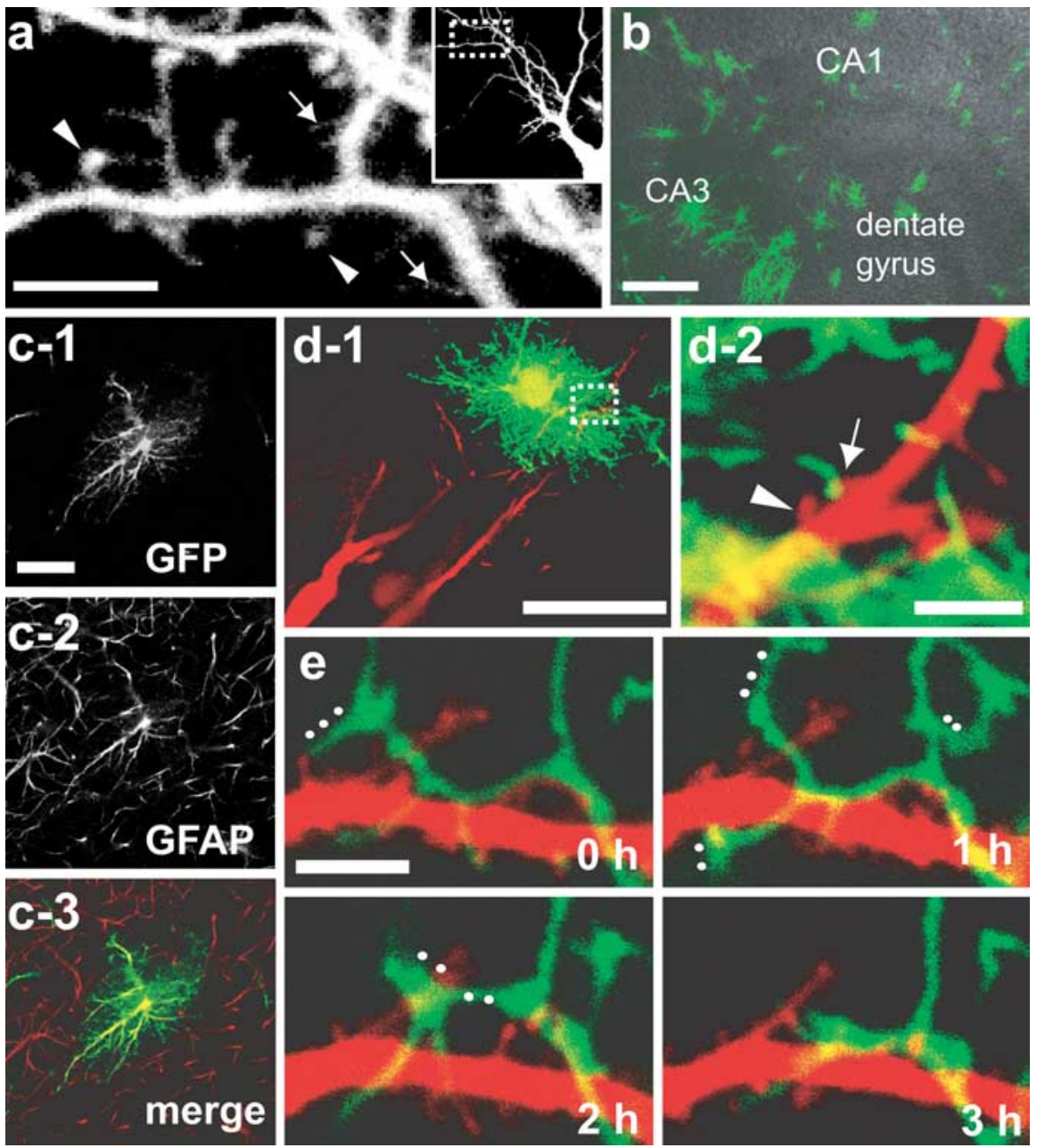

Figure 1. Simultaneous visualization of dendrites and astrocytic processes in hippocampal slice culture. $\boldsymbol{a}$, Dendritic protrusions visualized by rhodamine- dextran electroporation in an immature slice ( $5 \mathrm{~d}$ in vitro). Filopodia-like dendritic protrusions (arrows) and spines having bulbous heads (arrowheads) exist concurrently. The inset shows a lower-magnification view with a rectangle indicating the enlarged area. $\boldsymbol{b}, \boldsymbol{c}$, Morphology of GFP-positive astrocytes. Sparsely distributed GFP-expressing cells ( $\boldsymbol{b}$; green) extend thick protrusions with numerous fine processes on their surface (c-1) and show GFAP immunoreactivity $(\mathbf{c}-\mathbf{2}, \mathbf{c}-\mathbf{3})$, indicating their identity as astrocytes. Each image in $c$ is a single optical section. GFAP-positive processes extending from GFPnegative astrocytes show limited overlaps with the GFP-positive astrocyte. $\boldsymbol{d}$-1, Pyramidal neurons visualized by rhodaminedextran (red) in the vicinity of a GFP-positive astrocyte (green). $\boldsymbol{d}$-2, High-magnification view of the white rectangle in $\boldsymbol{d}$-1. Two adjacent dendritic protrusions are either with (arrows) or without (arrowheads) astrocytic contacts. $\boldsymbol{e}$, Multiple direct contacts between astrocytic processes (green) and dendritic protrusions (red). Rapid and extensive structural changes of astrocytic processes are evident. White dots indicate the astrocytic processes disappeared or changed their structure extensively in the following time frames (60 min intervals). Scale bars: $\boldsymbol{a}, 5 \mu \mathrm{m} ; \boldsymbol{b}, 200 \mu \mathrm{m} ; \boldsymbol{c}-1,20 \mu \mathrm{m} ; \boldsymbol{d}-\mathbf{1}, 50 \mu \mathrm{m} ; \boldsymbol{d}-\mathbf{2}, \boldsymbol{e}, 3 \mu \mathrm{m}$.

The spine index (see Figs. $4 e, 5 g$ ) was a proportion of the time frames belonging to $\mathrm{S}+\mathrm{A}$ or $\mathrm{S}$ to all the time frames in which the dendritic protrusion was present during a $5.5 \mathrm{~h}$ period of observations.

Statistical analysis included apical dendrites of both CA1 and CA3, because they did not show any significant difference in the aspects we analyzed in this study. To assess statistical significance, Student's $t$ test (see Figs. $3 g-i, 5 g$ ), Welch's $t$ test (see Figs. $3 f, 5 e, f$ ), and ANOVA with post hoc multiple comparisons by Tukey's test (see Fig. $4 d, e, 6 c, d$ ) were used. Data are given as mean \pm SEM. The number of slices (equals number of cells) analyzed were as follows: 11 for control experiments, 5 for mutant Racl experiments, and 5 for each experiment of the Fc applications.

Three-dimensional reconstruction and surface rendering was performed using IMARIS (Zeiss, Thornwood, NY) from two-photon images of multiple optical sections (nominal spatial resolution of $31 \mathrm{~nm}$ per pixel, 19 sections, and $z$-spacing of $0.2 \mu \mathrm{m}$ ). Outlines of astrocytic processes at multiple time points (see Fig. $5 a-3, b-3$ ) were extracted by binarizing the images with MetaMorph software.

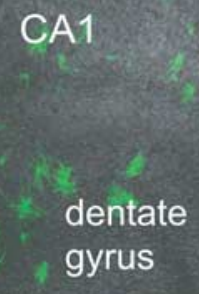

\section{Electron microscopy}

Slice cultures ( $6 \mathrm{~d}$ in culture) were fixed in $2.5 \%$ glutalaldehyde, $4 \%$ paraformaldehyde, and 0.1 M cacodylate buffer overnight. Serial thin sections were obtained in $55 \mathrm{~nm}$ thickness. Threedimensional reconstruction of images was performed by using the Reconstruct software (downloaded from Synapse Web, http://www.synapse-web.org/).

\section{Results}

Simultaneous time-lapse observation of dendritic protrusions and astrocytic processes

Pyramidal neuron dendrites filled with an inert fluorescent probe rhodamine-dextran (Fig. 1a) showed rapid protrusive activity in slices $<7 \mathrm{~d}$ in culture. More than $40 \%$ of dendritic protrusions showed a lifetime of $<4 \mathrm{~h}$, with drastic change of their shape and size. Rapid protrusive activity was suppressed in mature slices. Importantly, we noticed more heterogeneity in the lifetime of protrusions at early stages, which can be regulated by their interaction with other cellular components, such as axon terminals and astrocytic processes. Although the importance of axonfilopodia contacts has been demonstrated in previous imaging studies (Ziv and Smith, 1996), contribution of astrocytic processes in stabilizing dendritic protrusion has not been investigated extensively. Cre recombinase-dependent expression of GFP in a subset of astrocytes in tissue slices was achieved by combined infection of two recombinant adenoviruses (Fig. 1b). One was for the expression of Cre recombinase (Ad-Cre), and the second was for the expression of GFP under the CAG promoter with intervening loxP sites and stuffer sequences (Ad-loxP-GFP). Coinfection of the two adenoviruses initiated GFP expression in a small subset of cells, because the number of PFUs of Ad-Cre was adjusted to 1:10,000 of Ad-loxP-GFP. The majority of GFP-positive cells had several thick main protrusions with numerous fine processes on their surface. This morphology is consistent with astrocytes, and their identity was also confirmed by GFAP immunoreactivity (Fig. 1c). GFPpositive neurons and oligodendrocytes were few, because of the higher affinity of adenoviruses for astrocytes.

We could successfully identify contact sites between dye-filled dendritc protrusions and GFP-expressing astrocytes (Fig. 1d,e). In the subsequent analysis, contact sites were defined as overlapping pixels of two structural components within a single optical section. Images of contacting dendrites and astrocytes in figures are presented as either a single $z$-section or a projection of consecutive planes, depending on the obliqueness of dendrites and astrocytic processes. Astrocytic processes showed a tendency to rapidly change their shape and position (Hirrlinger et al., 2004; Benediktsson et al., 2005; Haber et al., 2006), frequently contacting with dendritic shafts and protrusions (Fig. 1e) (Haber et al., 

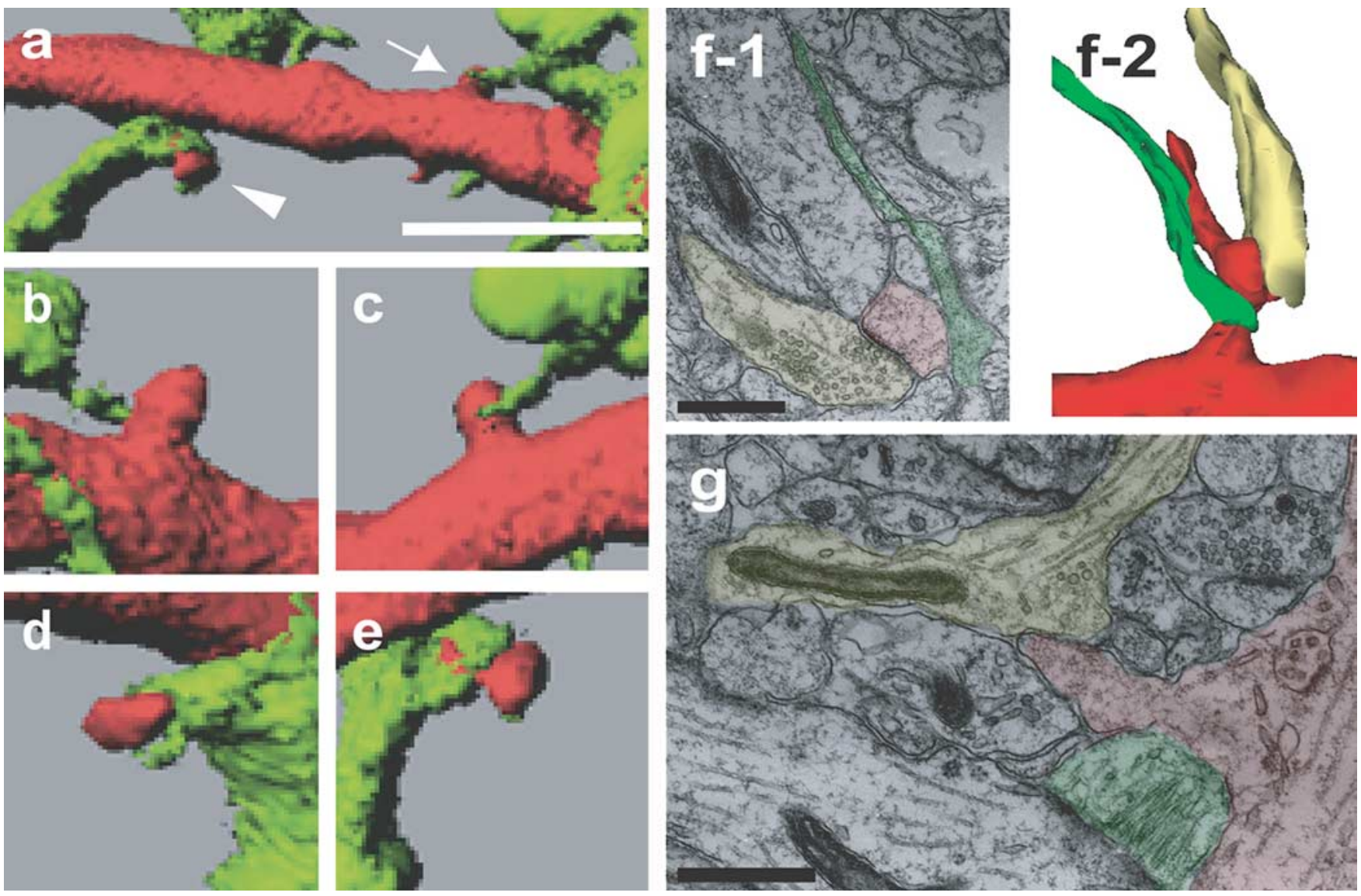

Figure 2. Morphology of contact sites between astrocytes and dendrites. $\boldsymbol{a}-\boldsymbol{e}$, Variable morphology of contact sites revealed by three-dimensional modeling of two-photon image stacks. Contact sites are either large enough to encircle the neck of dendritic protrusions (arrowhead in $\boldsymbol{a}$; enlarged in $\boldsymbol{d}$, $\boldsymbol{e}$ ) or confined to a small area (arrow in $\boldsymbol{a}$; enlarged in $\boldsymbol{b}, \boldsymbol{c}$ ). Higher-magnification views are from different angles (c and $\boldsymbol{e}$ are from the similar direction as in $\boldsymbol{a}$, and views from different angles are in $\boldsymbol{b}$ and $\boldsymbol{d}$ ). $\boldsymbol{f}$, Three-dimensional reconstruction of electron microscopic images from the area CA1 of a slice culture ( $6 \mathrm{~d}$ in vitro) illustrating contacts between a dendritic protrusion (red) and an axon terminal (yellow) and also between a dendritic protrusion and an astrocytic process ( $g r e e n ; f-2)$. A single micrograph containing the structure of a typical synaptic junction from the serial electron microscopic images is shown in $\boldsymbol{f}-1 . \boldsymbol{g}$, A single electron microscopic image from the area CA1 of a slice culture ( $6 \mathrm{~d}$ in vitro) showing contacts between a dendritic protrusion (red) and an astrocytic process (green). Although three-dimensioal reconstruction analysis identified physical association of the dendritic protrusion with axonal components (yellow), no prominent synaptic junctions could be identified. Scale bars: $\boldsymbol{a}, 5 \mu \mathrm{m} ; \boldsymbol{f}-\mathbf{1}, \boldsymbol{g}, 500 \mathrm{~nm}$.

2006). The overall morphology and position of dendritic shafts were relatively stable in the observation period of several hours. Detailed morphology of contact sites was evaluated by threedimensional modeling of two-photon image stacks. Dendritic protrusions were either surrounded by astrocytic processes or touched by their tips (Fig. $2 a-e$ ). Analysis of fixed tissue slices $<7$ $\mathrm{d}$ in culture revealed that GFP-positive immature astrocytes already had exclusive boundaries with few overlaps of GFAPpositive protrusions belonging to nearby GFP-negative cells (Fig. $1 c$; supplemental Fig. $1 a, b$, available at www.jneurosci.org as supplemental material) (Bushong et al., 2002, 2004). Therefore, it is less likely that dendritic protrusions in close vicinity of GFPlabeled astrocytes have additional contacts with unlabeled astrocytes. Retrospective analysis of imaged samples confirmed that the contact sites between dye-filled dendritic protrusions and GFP-positive astrocytes were located in the middle layer of tissue slices (supplemental Fig. 1c, available at www.jneurosci.org as supplemental material), where tissue organization of the normal neuropil was preserved without bundles of astrocytic processes (Benediktsson et al., 2005). Ultrastructural analysis confirmed the presence of astrocytic contacts with dendritic protrusions that already formed synaptic contacts at their base or neck (Fig. $2 f$ ) (Fiala et al., 1998). Astrocytic processes were not necessarily apposed to synaptic clefts but were associated with extrasynaptic domains of dendritic protrusions, suggesting specific roles of astrocytic contacts other than the neurotransmitter clearance. There also exist dendritic protrusions with astrocytic contacts but without any prominent postsynaptic specializations (Fig. $2 g$ ), suggesting early astrocytic contacts onto immature dendritic protrusions.

\section{Dendritic protrusions with astrocytic contacts show longer lifetime and higher tendency to form spines}

Repetitive two-photon scanning of tissue volumes containing both dendritic protrusions and astrocytic processes at intervals of $30 \mathrm{~min}$ and a duration of $5.5 \mathrm{~h}$ revealed multiple events of contacts between astrocytic processes and dendritic protrusions (Fig. $3 a-e)$. Time-lapse imaging with a higher sampling frequency confirmed the absence of multiple protrusive activities from the same dendritic position within $30 \mathrm{~min}$. The duration of contacts was variable in the range of $<30 \mathrm{~min}$ to $5.5 \mathrm{~h}$. Dendritic protrusions showed a tendency to be stabilized after contacts with astrocytes (Fig. 3a) but retracted when they failed to contact astrocytic processes (Fig. 3b). New dendritic protrusions experienced either prolonged or transient contacts with astrocytes (Fig. $3 c, e$ ). In both cases, dendritic protrusions with episodes of astrocytic contacts showed a tendency to persist longer. Systematic analysis of the relationship between contact events and stability of den- 


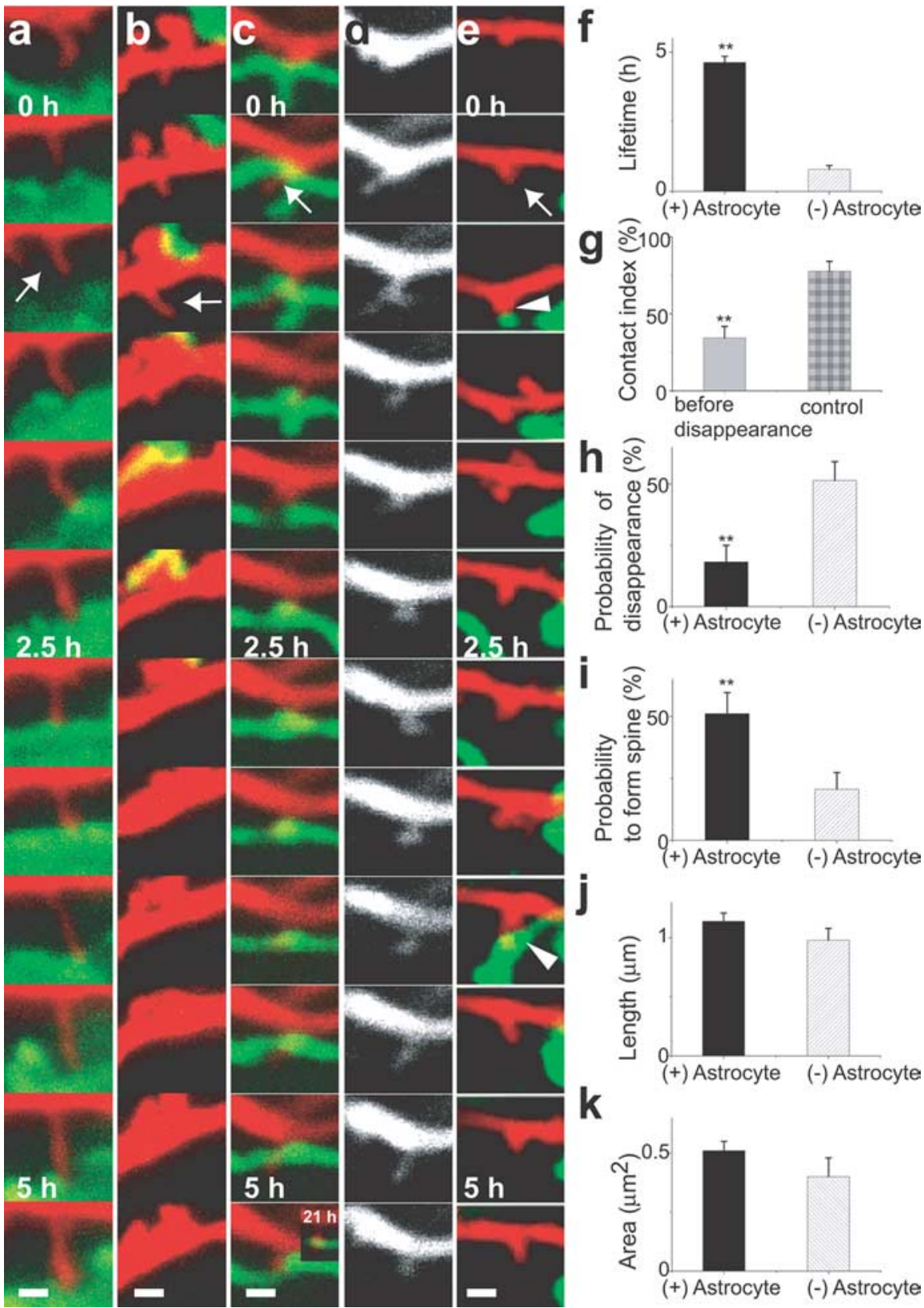

Figure 3. Enhanced lifetime and maturation of dendritic protrusions by astrocytic contacts. $\boldsymbol{a}-\boldsymbol{e}$, Fates of dendritic protrusions (red) with $(\boldsymbol{a}, \boldsymbol{c}, \boldsymbol{e}$, arrows) or without (b, arrow) astrocytic contacts (green). Dendritic protrusions persisted with astrocytic contacts $(\boldsymbol{a}, \boldsymbol{c}, \boldsymbol{e})$ but retracted without contacts $(\boldsymbol{b})$. New dendritic protrusions $(\boldsymbol{c}, \boldsymbol{e})$ showed either prolonged $(\boldsymbol{c})$ or transient $(\boldsymbol{e}$, arrowheads) astrocytic contacts. In both cases, dendritic protrusions were subsequently stabilized. The same dendritic protrusion is presented with different colors in c (red) and $\boldsymbol{d}$ (white). $\boldsymbol{f}$, Enhanced lifetime of dendritic protrusions with episodes of astrocytic contacts calculated from $5.5 \mathrm{~h}$ image sequences $[n(D P)=38 / 12$ (with $(+) /$ without $(-)$ Astrocyte, for all data in this legend]. $g$, Reduced contacts with astrocytes in the $1 \mathrm{~h}$ period before retraction of dendritic protrusions $[n(D P)=38]$. $\boldsymbol{h}, \boldsymbol{i}$, Astrocytic contacts reduce disappearance of dendritic protrusions $[n(D P)=33 / 30]$ and increase the probability to form spines $[n(D P)=32 / 30]$. $\boldsymbol{j}, \boldsymbol{k}$, Length and area of dendritic protrusions with or without astrocytic contacts $[n(D P)=260 / 142]$. All data are shown as mean \pm SEM. ${ }^{* *} p<0.01$. Scale bars, $1 \mu \mathrm{m}$. DP, Dendritic protrusion.

dritic protrusions revealed significantly a longer lifetime of dendritic protrusions with episodes of astrocytic contacts than those without contacts (Fig. $3 f$ ). Short-lived dendritic protrusions may have less contact simply because of their shorter residence time. However, this was not the case. Dendritic protrusions having a fate of disappearance showed a significantly lower tendency to contact astrocytes, even after normalization of their residence time (Fig. $3 g$ ). Furthermore, dendritic protrusions without astrocytic contacts disappeared with significantly higher probability than those with astrocytes (Fig. 3h). Astrocyte-positive and -negative dendritic protrusions were sampled from the common dendritic segments with a similar distance from astrocytic structures. Therefore, the selective stabilization is attributable to neither a difference in the general maturity of neurons nor a difference in the concentration of astrocytederived diffusible factors. Another important feature of dendritic protrusions with astrocytic contacts was their tendency to form spines, which can be maintained for a prolonged period (Fig. 3c). Astrocytic contacts significantly increased the tendency of transition to spines from other categories of dendritic protrusions, including filopodia-like and stubby protrusions (Fig. 3i). Mean length and area of dendritic protrusions were similar regardless of astrocytic contacts (Fig. 3j,k), suggesting that astrocytic contacts influence the stability of dendritic protrusions independent of their size.

\section{Regulated motility of astrocytic processes and heterogeneity of contact events}

Two dynamic properties were evident in contact events. First, astrocytic processes, which showed higher motility than dendritic protrusions, tended to elongate or shrink toward specific destinations where they contacted dendritic protrusions. The destination was either the position where dendritic protrusions had already existed (Fig. $4 a$ ) or the position where their tips finally arrived (Fig. 4b). This motility indicates the presence of specific spatial signals, such as chemoattractants including glutamate released from presynaptic sites (Cornell-Bell et al., 1990; Portera-Cailliau et al., 2003). Second, the contact duration could be clearly classified into either short $(<1 \mathrm{~h})$ or long $(>5 \mathrm{~h})$ duration (Fig. $4 c)$. The lifetime of dendritic protrusions showed a positive correlation with both contact duration and the presence of contacts (Fig. 4d). In turn, the tendency to form spines was dependent mainly on whether the contact was maintained for a prolonged period or not (Fig. 4e). These results indicate that signals activated by transient astrocytic contacts are sufficient for stabilization of dendritic protrusions, but their subsequent maturation requires other signaling mechanisms activated by prolonged interactions.

Inhibition of astrocytic motility by Racl affects maturation of dendritic protrusions

Does astrocytic contact to dendritic protrusions indeed have causal effects on their subsequent fate? To address this issue, we attempted to chronically suppress astrocytic motility by manip- 


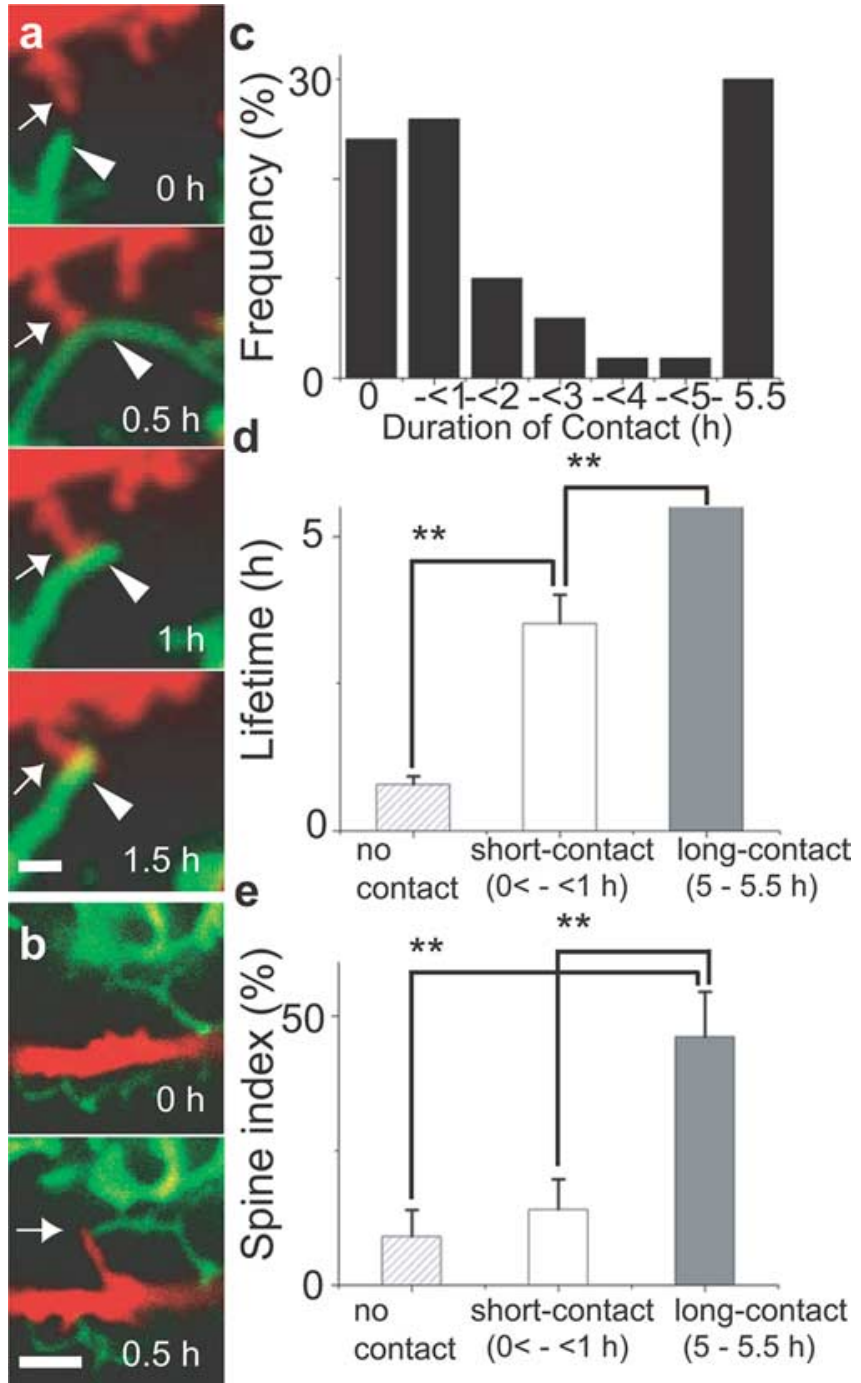

Figure 4. Multiple modes of interaction between astrocytes and dendrites. $\boldsymbol{a}$, Rapid motility of an astrocytic process (green) and its contact with a dendritic protrusion (red). A dendritic protrusion (arrow) started to contact the side of an extending astrocytic process (arrowhead; $0.5 \mathrm{~h}$ ). This astrocytic process retracted to the point of the contact $(1 \mathrm{~h})$ and was subsequently stabilized (1.5 h). $\boldsymbol{b}$, Coordinated protrusive activity of dendrites (red) and astrocytes (green). The tips of a new filopodia-like dendritic protrusion and an astrocytic process reached the same destination (arrow). c, Distribution of contact duration of each dendritic protrusion. Contact duration can be classified into three groups: with no contacts, with short contacts ( $<1 \mathrm{~h}$ ), and with prolonged contacts $(>5 \mathrm{~h}) . \boldsymbol{d}, \boldsymbol{e}$, Lifetimes of dendritic protrusions $(\boldsymbol{d})$ and the tendency to form spines $(\boldsymbol{e})$ among groups with different duration of astrocytic contacts [ $n$ (dendritic protrusion) $=12$ (no contact), 13 (short-contact), 15 (long-contact)]. All data are shown as mean \pm SEM. ${ }^{* *} p<0.01$. Scale bars: $\boldsymbol{a}, 1 \mu \mathrm{m} ; \boldsymbol{b}, 3 \mu \mathrm{m}$.

ulating a Rac1-dependent signaling pathway. Both mutant Rac1 (N17) (Ridley et al., 1992) and GFP were expressed in astrocytes for $48 \mathrm{~h}$ using a bicistronic recombinant adenoviral vector at 4-7 $\mathrm{d}$ in culture (Fig. $5 a$ ). Because recombinant adenoviruses show higher affinity for astrocytes, we detected predominant expression of GFP in astrocytes, whereas the expression in neurons was undetectable. Expression of mutant Racl stabilized astrocytic processes, without changing the motility of dendritic protrusions (Fig. $5 a, b, e$ ). In this situation, contact events initiated by astrocytic motility were chronically suppressed (Fig. 5a1-a3, b1-b3). Importantly, this manipulation induced significantly longer, filopodia-like dendritic protrusions (Fig. 5c,d,f,g). An undetectable level of both GFP and mutant Racl might be expressed in neurons and affect spine morphology. However, studies in dissociated hippocampal culture confirmed negligible effects of neuronal expression of mutant Racl at the lower level on the morphology of dendritic spines (supplemental Fig. 2, available at www.jneurosci.org as supplemental material). It should also be emphasized that general motility and lifetimes of dendritic protrusion in slices infected with Racl adenoviruses were preserved (Fig. $5 e, h$ ). This observation again argues against direct effects of inhibiting neuronal Rac1, which has been reported to destabilize dendritic protrusions (Tashiro and Yuste, 2004). Thus, we conclude that a chronic blockade of Rac1-dependent astrocytic motility reduces maturation of dendritic protrusions.

\section{Perturbation of ephrin/Eph signaling affects lifetime of dendritic protrusions}

We next analyzed instructive roles of astrocytic contacts on stability of dendritic protrusions by acute interference of a cellsurface ligand-receptor system. Activation of EphA4 receptors on dendritic spines by astrocytic ephrin-A3 maintains normal spine morphology in the hippocampus (Murai et al., 2003; Martínez and Soriano, 2005). EphA4/Fc (chimeric molecules of EphA4 and IgG Fc portion) will inhibit activation of endogenous EphA receptors by occupying ephrin-A ligands on the surface of astrocytic processes. Ephrin-A3/Fc can induce constitutive EphA receptor activation independent of contact events with astrocytes. Thus, both EphA4/Fc and ephrin-A3/Fc treatments can perturb the contact-dependent ephrin/Eph signaling by astrocytes. Consistent with this notion, in the presence of EphA4/Fc or ephrin-A3/Fc, dendritic protrusions having episodes of astrocytic contacts showed a significantly shorter lifetime (Fig. $6 a-c$ ). These treatments did not alter the lifetime of dendritic protrusions without astrocytic contacts. Acute application of EphA4/Fc and ephrin-A3/Fc may be less effective in destabilizing preexisting dendritic protrusions, because they may have already experienced astrocytic contacts before imaging sessions. Indeed, the effects of both EphA4/Fc and ephrin-A3/Fc were more prominent in newly generated dendritic protrusions (Fig. $6 d$ ). Thus, acute interference of ephrin/Eph signaling preferentially destabilized newly generated dendritic protrusions with episodes of astrocytic contact. There was a downward trend in contact duration of newly formed dendritic protrusions in the presence of either EphA4/Fc or ephrin-A3/Fc (Fig. 6e). The probability of transition into spines after astrocytic contacts was not significantly reduced in the presence of EphA4/Fc or ephrin-A3/Fc (Fig. 6f). This measurement may underestimate the effects of EphA4/Fc and ephrin$\mathrm{A} 3 / \mathrm{Fc}$ on spine maturation, because these treatments induce immediate destabilization of new protrusions, resulting in the progressive elimination of young protrusions from the cohort. This sampling bias may account for the relatively high probability of transition into spines in the presence of EphA4/Fc or ephrinA3/Fc. Nevertheless, the results indicate less prominent effects of perturbing ephrin/Eph signaling in both contact duration and morphological transition of stable dendritic protrusions into spines. We conclude that acute interference of ephrin/Eph signaling mainly affects astrocyte-dependent stabilization of newly formed dendritic protrusions.

\section{Discussion}

In this study, we presented evidences of frequent contact events between astrocytic processes and dendritic protrusions in developing hippocampal tissue slices. Astrocytic contacts showed positive correlation with both lifetime and morphological maturation of dendritic protrusions. The causal relationship between 

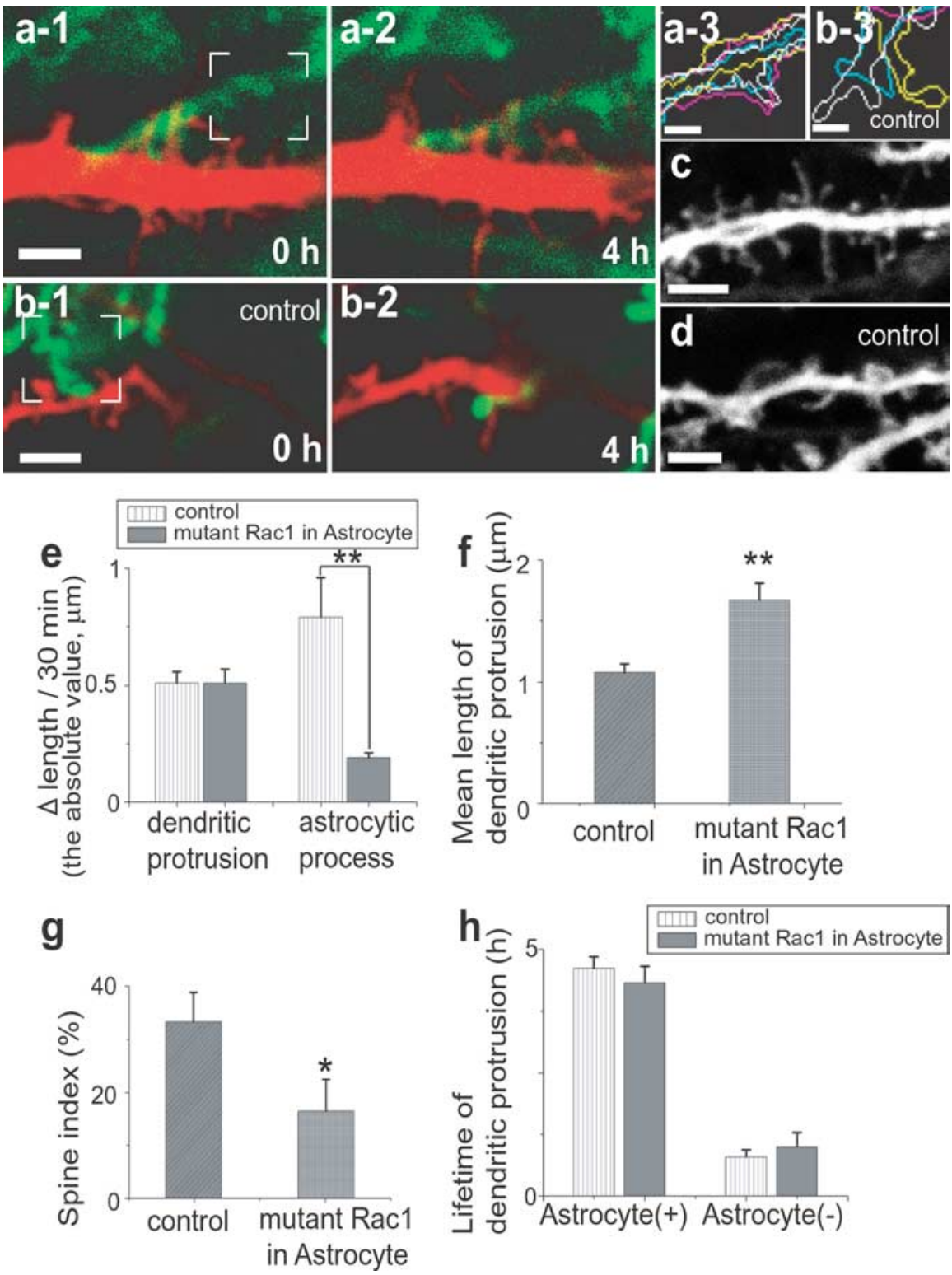

Figure 5. Effect of suppressing astrocytic motility by mutant Rac1. $\boldsymbol{a}, \boldsymbol{b}$, Reduced motility of astrocytes expressing mutant Rac1 (green; $\boldsymbol{a}-\mathbf{1}, \boldsymbol{a}-\mathbf{2}$ ) in the vicinity of dendrites (red). Control astrocytes show higher motility (green; $\boldsymbol{b}-\mathbf{1}, \boldsymbol{b}-\mathbf{2}$ ). The difference is evident by overlaying outlines of astrocytes at $0,1.5,2.5$, and $4 \mathrm{~h}$ (yellow, blue, white, and pink lines, respectively, in $\boldsymbol{a}-\mathbf{3}$ and $\boldsymbol{b}-\mathbf{3}$ ) within the white rectangles in $\boldsymbol{a}-\mathbf{1}$ and $\boldsymbol{b}-\mathbf{1}$. $\boldsymbol{c}, \boldsymbol{d}$, Induction of long, filopodia-like dendritic protrusions (c) in neurons by overexpression of mutant Rac1 selectively in astrocytes. $\boldsymbol{e}$, Average difference of protrusion length per imaging frame for both dendrites and astrocytes $[n(D P)=13 / 49, n$ (astrocytic process) $=6 / 32$, mutant Rac1/control, for all data in this legend].f, $\boldsymbol{g}$, Length $(\boldsymbol{f})$ and the tendency to form spines $(\boldsymbol{g})$ of dendritic protrusions with or without astrocytic expression of mutant $\operatorname{Rac} 1[n(D P)=45 / 36$, 50/36]. $\boldsymbol{h}$, Unaltered lifetimes of dendritic protrusions with or without astrocytic expression of mutant Rac1. All data are shown as mean \pm SEM. ${ }^{*} p<0.05 ;{ }^{* *} p<0.01$. Scale bars: $\boldsymbol{a}-\mathbf{1}, \boldsymbol{b}-\mathbf{1}, \boldsymbol{c}, \boldsymbol{d}, 3 \mu \mathrm{m} ; \boldsymbol{a}-\mathbf{3}, \boldsymbol{b}-\mathbf{3}, 1 \mu \mathrm{m}$. DP, Dendritic protrusion.

astrocytic contacts and stabilization/maturation of dendritic protrusions was supported by two experimental evidences. First, inhibition of astrocytic motility by perturbation of Rac1-dependent signaling results in development of abnormal, thin morphology of dendritic protrusions. Second, interference of ephrin/Eph signaling specifically reduced the lifetime of newly generated dendritic protrusions with astrocytic contacts. Thus, we conclude that direct astrocytic contacts have instructive roles in subsequent stabilization and maturation of immature dendritic protrusions.

An important aspect of the signaling system reported here is its spatial selectivity. Adjacent dendritic protrusions can have different fates, if one receives astrocytic contacts and the other does not. Previous characterization of filopodia dynamics in hippocampal pyramidal neurons indicated that their motility fell into two major classes, short-lived ( $<20 \mathrm{~min})$ and longlived $(>100 \mathrm{~min}$ ) groups (Dailey and Smith, 1996), which may be related to their differential association with astrocytes. Astrocytic processes elongate and retract rapidly with intervening pauses at specific positions where dendritic protrusions frequently reside. This motility is analogous to that of dendritic filopodia, which are thought to be involved in spatial sampling of their targets (Dailey and Smith, 1996; Ziv and Smith, 1996; PorteraCailliau et al., 2003; Yuste and Bonhoeffer, 2004). The ability of astrocytes to identify appropriate targets may exceed that of dendritic filopodia, because astrocytic protrusive activity is more extensive and rapid. Astrocytic protrusive activity is complex in their morphology, including frequent branching, ramification, and formation of the veil-like structure. It is likely that these multiple motile behaviors are precisely controlled by specific signaling pathways. Indeed, we observed directional protrusive activity of astrocytic processes toward specific points where dendritic structure is present. It is likely that both the direction and the extent of astrocytic protrusive activity are regulated by environmental cues including diffusible factors from presynaptic and postsynaptic structures, resulting in specific interactions of astrocytes with a specific subset of nascent synapses. Previous studies showed glutamate-dependent filopodial formation both from astrocytes and neurons, suggesting participation of presynaptically released glutamate in their protrusive activities. Glutamate application or placement of pyramidal neurons onto cultured astrocytes increases the number of filopodia-like protrusions on the surface of cultured astrocytes (Cornell-Bell et al., 1990). Similarly, a subset of dendritic filopodia elongate after focal application of glutamate (Portera-Cailliau et al., 2003). The intracellular signaling pathway involved in directional motility of astrocytic processes has not been identified. A previous study on polarized motility of astrocytes in vitro revealed the importance of the Cdc42-glycogen synthase kinase $3 \beta$-adenomatous polyposis coli pathway (Etienne-Manneville and Hall, 2003). However, this system operates through the Apcdependent reorganization of microtubules, which are less likely to be present in thin processes of astrocytes. A recent time-lapse imaging of astrocytes in tissue slices showed significant reduction of their motility in the presence of cytochalasin-D, suggesting its actin dependence (Haber et al., 2006). At least our time-lapse imaging study suggests involvement of Rac1-dependent signaling 
in the regulation of motile behavior of astrocytic processes in the vicinity of nascent synapses.

Axon-dendrite adhesion and subsequent bidirectional signaling have been demonstrated to be essential in initial synaptic differentiation. How does the axondendrite interaction cooperate with astrocytic contact events? The transient nature of astrocytic contact suggests its instructive role in an early phase of spine formation, even before formation of synaptic junctions (Fig. $2 g$ ). In this sense, astrocytemediated stabilization of dendritic protrusions may increase their lifetime, resulting in both promotion of their initial contact with axons and subsequent formation of the synaptic junctional complex. This idea is consistent with a recent study of in vivo two-photon imaging with serial section electron microscopy, which revealed that more than half of new spines lack synaptic junctions (Knott et al., 2006). In turn, our ultrastructural analysis revealed the presence of dendritic protrusions with both astrocytic contacts and postsynaptic specialization, suggesting roles of astrocytic contacts on maturation of pre-established synapses. Because the distribution of contact duration showed clearly two peaks, the mode of astrocytic contacts may be multiple, either transient stabilization of motile filopodia or induction of spine maturation by prolonged association. Consistent with this idea, $\sim 40 \%$ of contact

Figure 6. Effect of acute perturbation of ephrin/Eph signaling. $\boldsymbol{a}, \boldsymbol{b}$, Time-lapse imaging of dendrites (red) and astrocytic processes (green) in the presence of either EphA4/Fc (a) or ephrin- $\mathrm{A} 3 / \mathrm{Fc}(\boldsymbol{b})$. Reduced stability of dendritic protrusions (arrows) is evident in both treatments. c, Lifetime of dendritic protrusions with astrocytic contact episodes [( + ) Astrocyte] is altered in the presence of EphA4/Fc or ephrin-A3/Fc. In contrast, those without contacts [(-) Astrocyte] show no significant difference $[n(D P)=17 / 3$ (with $(+) /$ without $(-)$ Astrocyte, in the presence of EphA4/Fc), 18/5 (in the presence of ephrinA3/Fc), 27/7 (in the presence of $\mathrm{Fc}$ alone), 38/12 (control)]. $\boldsymbol{d}$, Selective reduction in the lifetime of newly generated dendritic protrusions by either EphA4/Fc or ephrin- $\mathrm{A} / \mathrm{Fc}$ treatment $[n(D P)=7 / 10$ (new DP/preexisting $D P$, in the presence of EphA4/Fc), 5/13 (in the presence of ephrin-A3/ $\mathrm{Fc}$ ), 12/15 (in the presence of $\mathrm{Fc}$ alone), 7/31 (control)]. $\boldsymbol{e}$, Downward trend in contact duration of newly generated dendritic protrusions treated with either EphA4/Fc or ephrin$A 3 / F c[n(D P)=7 / 10$ (new DP/preexisting DP, in the presence of EphA4/Fc), $5 / 13$ (in the presence of ephrinA3/Fc), 12/15 (in the presence of $F($ alone), $7 / 31$ (control)]. $f$, Probability of dendritic protrusions to form spines in the presence of EphA4/Fc or ephrin-A3/Fc $[n(D P)=14 / 4$ (with (+)/without (-) Astrocyte, in the presence of EphA4/Fc), 15/12 (in the presence of ephrinA3/Fc), 15/10 (in the presence of $\mathrm{Fc}$ alone), 32/30 (control)]. All data are shown as mean \pm SEM. ${ }^{*} p<0.05 ; * * p<$ 0.01. Scale bar, $1 \mu \mathrm{m}$. DP, Dendritic protrusion.
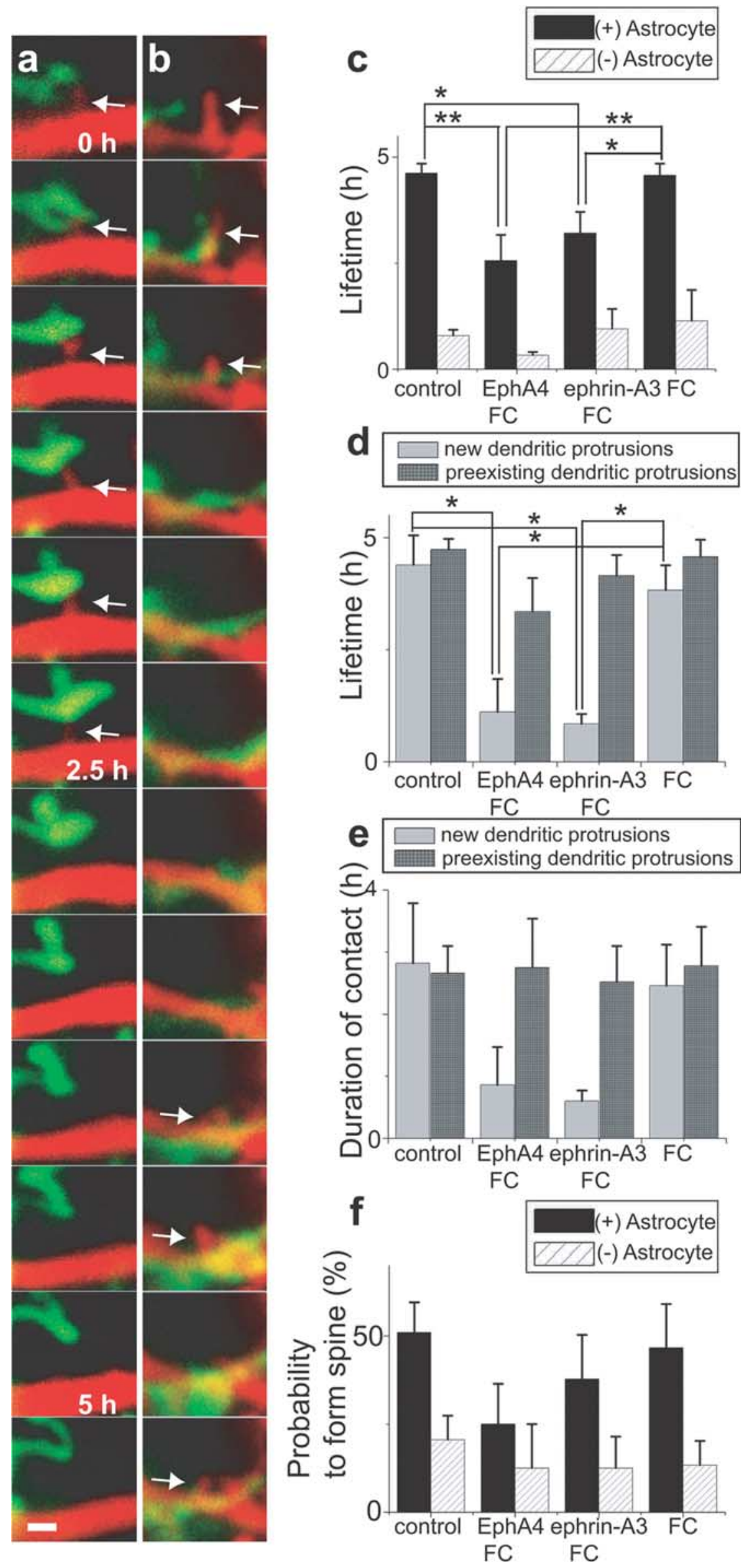
events lasted $>5 \mathrm{~h}$, and the dendritic protrusions in this category showed both a longer lifetime and a higher tendency of transformation into spines (Fig. 4). It will be reasonable to speculate that astrocytic contacts operate in both stabilization of newly formed dendritic protrusions before their formation of synaptic contacts and later maturation of spines. It should also be emphasized that the axonal structure can potentially regulate the interaction between dendrites and astrocytes even in the absence of direct contacts. As discussed previously, presynaptically released glutamate may initiate structural change of both dendrites and astrocytes. It remains to be demonstrated that astrocyte-mediated stabilization of dendritic protrusions takes place independent of diffusible factors released from axonal components.

We observed the transformation of dendritic protrusions into thin and long processes after chronic inhibition of Rac1dependent astrocytic motility. In the absence of motile astrocytic processes, the probability of contacts between dendrites and astrocytes will be mostly reduced. Therefore, it is possible that the dendritic protrusions with aberrant morphology are those developed to form stable synaptic junctions without support of previous astrocytic contacts. Another possibility is that longer dendritic protrusions are generated because of the increased distance between dendritic shafts and astrocytic processes after suppression of astrocytic motility. Only longer dendritic filopodia that managed to contact astrocytes can be subsequently stabilized and survive. Consistent with this idea, dendritic protrusions with astrocytic contacts still show longer lifetime in slice preparations infected with mutant Rac1-expressing adenoviruses (Fig. 5h), indicating that mutant Racl expression in astrocytes does not abolish their stabilizing effects on dendritic protrusions. Although we are short of experimental evidences discriminating these two possible models at present, both indicate essential roles of astrocytic motility in normal morphogenesis of dendritic protrusions.

Our perturbation experiments of the ephrin/Eph system indicate its involvement in the contact-dependent signaling between astrocytes and dendrites. Astrocytic ephrin-A3 induces activation of EphA4 receptors on dendritic spines and is essential in the maintenance of normal spine morphology (Murai et al., 2003). It has been reported that acute administration of ephrin-A3/Fc results in shrinkage of preexisting dendritic spines in mature hippocampal CA1 pyramidal neurons. In turn, acute EphA4/Fc administration induces disorganization of spine morphology. The effect of EphA/Fc may be dependent on its duration of application, because chronic presence of EphA5/Fc in vivo by using transgenic overexpression results in reduction of the density of synaptic terminals in the hippocampus (Martínez et al., 2005). Our perturbation experiment using ephrin-A3/Fc is mostly consistent with its previously reported inhibitory effects on filopo$\mathrm{dia} /$ spine morphogenesis. Although application of ephrin-A3/Fc in the adult CA1 slices was effective in retraction of preexisting spines, our perturbation study revealed more prominent effects on newly generated dendritic protrusions. This difference may be explained by more abundant expression of cell adhesion molecules, such as $\mathrm{N}$-cadherin, in early developmental stages (Elste and Benson, 2006). The presence of strong mechanical adhesion of dendritic protrusions to either axons or astrocytes may support their ephrin/Eph-independent stabilization. In addition to ephrin-A3/Fc, EphA4/Fc was also effective in destabilization of newly generated dendritic protrusions. Because EphA4/Fc binds and masks ephrin ligands present on the surface of astrocytes, direct astrocytic contacts no longer initiate signaling events within the dendritic cytoplasm. Ephrin-Eph interaction activates guanine-nucleotide exchange factors, such as Ephexin (Shamah et al., 2001) and Tiam1 (Tanaka et al., 2004), and leads to reorganization of the actin cytoskeleton through modulation of Rho GTPases (Tashiro and Yuste, 2004; Redmond and Ghosh, 2001). Because Ephexin activity in the growth cone has been shown to promote the growth cone collapse through the enhancement of RhoA activation (Shamah et al., 2001), similar structural stabilization may play a role in maintenance of contacts between dendritic protrusions and astrocytes. Thus, both constitutive activation of Eph receptors by ephrin-A3/Fc and elimination of ephrin/ Eph signaling by EphA4/Fc interfere with proper signaling events triggered by astrocytic contacts, resulting in destabilization of newly generated dendritic protrusions.

Does similar contact-dependent signaling between astrocytes and dendritic spines operate in the adult brain? Half of excitatory synapses in the hippocampus are associated with astrocytic processes, and the remaining synapses also have astrocytic processes in their vicinity. Although in vivo imaging revealed limited motility of astrocytic processes (Nimmerjahn et al., 2005), neuronal activity may upregulate their motility. Indeed, a previous study reported synaptic recruitment of astrocytic processes in longterm potentiation (Wenzel et al., 1991). Two-photon imaging of multiple neural components in combination with manipulations of synaptic efficacy will reveal whether the contact-dependent, local signaling system described here also functions in the mature neuron-glia network.

\section{References}

Beattie EC, Stellwagen D, Morishita W, Bresnahan JC, Ha BK, Von Zastrow M, Beattie MS, Malenka RC (2002) Control of synaptic strength by glial TNF $\alpha$. Science 295:2282-2285.

Benediktsson AM, Schachtele SJ, Green SH, Dailey ME (2005) Ballistic labeling and dynamic imaging of astrocytes in organotypic hippocampal slice cultures. J Neurosci Methods 141:41-53.

Blondel O, Collin C, McCarran WJ, Zhu S, Zamostiano R, Gozes I, Brenneman DE, McKay RD (2000) A glia-derived signal regulating neuronal differentiation. J Neurosci 20:8012-8020.

Bushong EA, Martone ME, Jones YZ, Ellisman MH (2002) Protoplasmic astrocytes in CA1 stratum radiatum occupy separate anatomical domains. J Neurosci 22:183-192.

Bushong EA, Martone ME, Ellisman MH (2004) Maturation of astrocytic morphology and the establishment of astrocyte domains during postnatal hippocampal development. Int J Dev Neurosci 22:73-86.

Christopherson KS, Ullian EM, Stokes CC, Mullowney CE, Hell JW, Agah A, Lawler J, Mosher DF, Bornstein P, Barres BA (2005) Thrombospondins are astrocyte-secreted proteins that promote CNS synaptogenesis. Cell 120:421-433.

Cornell-Bell AH, Thomas PG, Smith SJ (1990) The excitatory neurotransmitter glutamate causes filopodia formation in cultured hippocampal astrocytes. Glia 3:322-334.

Dailey ME, Smith SJ (1996) The dynamics of dendritic structure in developing hippocampal slices. J Neurosci 16:2983-2994.

Elste AM, Benson DL (2006) Structural basis for developmentally regulated changes in cadherin function at synapses. J Comp Neurol 495:324-335.

Etienne-Manneville S, Hall A (2003) Cdc42 regulates GSK-3b and adenomatous polyposis coli to control cell polarity. Nature 42:753-756.

Fiala JC, Feinberg M, Popov V, Harris KM (1998) Synaptogenesis via dendritic filopodia in developing hippocampal area CA1. J Neurosci 18:8900-8911.

Haas K, Sin WC, Javaherian A, Li Z, Cline HT (2001) Single-cell electroporation for gene transfer in vivo. Neuron 29:583-591.

Haber M, Zhou L, Murai KK (2006) Cooperative astrocyte and dendritic spine dynamics at hippocampal excitatory synapses. J Neurosci 26:8881-8891.

Hama H, Hara C, Yamaguchi K, Miyawaki A (2004) PKC signaling mediates global enhancement of excitatory synaptogenesis in neurons triggered by local contact with astrocytes. Neuron 41:405-415.

Hansson E, Rönnbäck L (2003) Glial neuronal signaling in the central nervous system. FASEB J 17:341-348. 
Haydon PG (2001) Glia: listening and talking to the synapse. Nat Rev Neurosci 2:185-193.

Hirrlinger J, Hülsmann S, Kirchhoff F (2004) Astroglial processes show spontaneous motility at active synaptic terminals in situ. Eur J Neurosci 20:2235-2239.

Knott GW, Holtmaat A, Wilbrecht L, Welker E, Svoboda K (2006) Spine growth precedes synapse formation in the adult neocortex in vivo. Nat Neurosci 9:1117-1124.

Martínez A, Soriano E (2005) Functions of ephrin/Eph interactions in the development of nervous system: emphasis on the hippocampal system. Brain Res Rev 49:211-226.

Martínez A, Otal R, Sieber BA, Ibanez C, Soriano E (2005) Disruption of ephrin-A/EphA binding alters synaptogenesis and neural connectivity in the hippocampus. Neuroscience 135:451-461.

Mauch DH, Nägler K, Schumacher S, Göritz C, Müller EC, Otto A, Pfrieger FW (2001) CNS synaptogenesis promoted by glia-derived cholesterol. Science 294:1354-1357.

Murai KK, Nguyen LN, Irie F, Yamaguchi Y, Pasquale EB (2003) Control of hippocampal dendritic spine morphology through ephrin-A3/EphA4 signaling. Nat Neurosci 6:153-160.

Nimmerjahn A, Kirchhoff F, Helmchen F (2005) Resting microglial cells are highly dynamic surveillants of brain parenchyma in vivo. Science 308:1314-1318

Okabe S, Kim HD, Miwa A, Kuriu T, Okado H (1999) Continual remodeling of postsynaptic density and its regulation by synaptic activity. Nat Neurosci 2:804-811.

Portera-Cailliau C, Pan DT, Yuste R (2003) Activity-regulated dynamic behavior of early dendritic protrusions: evidence for different types of dendritic filopodia. J Neurosci 23:7129-7142.

Redmond L, Ghosh A (2001) The role of notch and Rho GTPase signaling in the control of dendritic development. Curr Opin Neurobiol 11:111-117.

Ridley AJ, Paterson HF, Johnston CL, Diekmann D, Hall A (1992) The small GTP-binding protein rac regulates growth factor-induced membrane ruffling. Cell 70:401-410.
Schipke CG, Kettenmann H (2004) Astrocyte responses to neuronal activity. Glia 47:226-232.

Shamah SM, Lin MZ, Goldberg JL, Estrach S, Sahin M, Hu L, Bazalakova M, Neve RL, Corfas G, Debant A, Greenberg ME (2001) EphA receptors regulate growth cone dynamics through the novel guanine nucleotide exchange factor ephexin. Cell 105:233-244.

Slezak M, Pfrieger FW (2003) New roles for astrocytes: regulation of CNS synaptogenesis. Trends Neurosci 26:531-535.

Stoppini L, Buchs PA, Muller D (1991) A simple method for organotypic cultures of nervous tissue. J Neurosci Methods 37:173-182.

Tanaka M, Ohashi R, Nakamura R, Shinmura K, Kamo T, Sakai R, Sugimura H (2004) Tiaml mediates neurite outgrowth induced by ephrin-B1 and EphA2. EMBO J 23:1075-1088.

Tashiro A, Yuste R (2004) Regulation of dendritic spine motility and stability by Racl and Rho kinase: evidence for two forms of spine motility. Mol Cell Neurosci 26:429-440.

Ullian EM, Sapperstein SK, Christopherson KS, Barres BA (2001) Control of synapse number by glia. Science 291:657-660.

Umeda T, Ebihara T, Okabe S (2005) Simultaneous observation of stably associated presynaptic varicosities and postsynaptic spines: morphological alterations of CA3-CA1 synapses in hippocampal slice cultures. Mol Cell Neurosci 28:264-274.

Ventura R, Harris KM (1999) Three-dimensional relationships between hippocampal synapses and astrocytes. J Neurosci 19:6897-6906.

Wang X, Lou N, Xu Q, Tian GF, Peng WG, Han X, Kang J, Takano T, Nedergaard M (2006) Astrocytic $\mathrm{Ca}^{2+}$ signaling evoked by sensory stimulation in vivo. Nat Neurosci 9:816-823.

Wenzel J, Lammert G, Meyer U, Krug M (1991) The influence of long-term potentiation on the spatial relationship between astrocyte processes and potentiated synapses in the dentate gyrus neuropil of rat brain. Brain Res 560:122-131.

Yuste R, Bonhoeffer T (2004) Genesis of dendritic spines: insights from ultrastructural and imaging studies. Nat Rev Neurosci 5:24-34.

Ziv NE, Smith SJ (1996) Evidence for a role of dendritic filopodia in synaptogenesis and spine formation. Neuron 17:91-102. 